\title{
The effects of phosphatidylserine supplementation on cognitive functioning prior and following an acute bout of resistance training in young males
}

\author{
Adam Parker ${ }^{1 *}$, Josh Gordon ${ }^{1}$, Aaron Thornton ${ }^{1}$, John Lubker ${ }^{1}$, Michelle Bartlett ${ }^{1}$, Ralf Jäger $^{2}$, Martin Purpura ${ }^{2}$,
} Mike Bird ${ }^{3}$, Jonathan Oliver ${ }^{3}$, Sunday Simbo ${ }^{3}$, Chris Rasmussen ${ }^{3}$, Richard B Kreider ${ }^{3}$

From International Society of Sports Nutrition: 7th Annual ISSN Conference and Expo

Clearwater Beach, FL, USA. 24-26 June 2010

\section{Background}

Making quick decisions and reducing the amount of errors at the beginning of a competition are crucial to the success in team sports and individual events. Phosphatidylserine (PS) has been shown to reduce stress and increase performance in runners, cyclists and golfers. A randomized, double-blind, placebo-controlled, cross-over pilot study was performed to evaluate the effect of PS supplementation on cognitive function prior to and following an acute bout of resistance training in 18 males aged 18-30.

\section{Methods}

During the first testing session, subjects were familiarized with the serial subtraction test (SST) and performed 1 repetition maximum (1RM) lifts in the smith machine squat (SQ), leg press (LP), and leg extension (LE). Subjects consumed PS (400 mg/day, SerinAid, Chemi Nutra) or placebo in a random, cross-over design for 14 days, with no washout period between supplementation. Following supplementation, subjects performed 5 sets of 10 repetitions at $70 \%$ of their $1 \mathrm{RM}$ on SQ, LP, and LE. SST was measured prior to exercise (PRE) and 5 (5POST) and 60 (60POST) minutes after exercise.

\section{Results}

PS supplementation significantly reduced the time needed for a correct calculation by $19.8 \%(1.27 \mathrm{~s}$ per calculation; Placebo: 6.4 s, PS $5.13 \mathrm{~s} ; \mathrm{p}=0.001)$, and

'Department of Sport and Exercise Science, West Texas A\&M University, Canyon, TX, 79016, USA

Full list of author information is available at the end of the article reduced the total amount of errors by 33\% (PRE: Placebo: 27, PS: $18, \mathrm{p}=0.18$ ) at PRE compared to placebo. Exercise significantly improved SST time $(\mathrm{p}=0.03)$. PS did not improve SST compared to placebo post exercise.

\section{Conclusion}

PS supplementation significantly increased cognitive function prior to exercise. Improved cognitive function could benefit athletes and non-athletes alike. Further research is warranted to determine the effects of varying dosages and duration of PS supplementation on cognitive function during exercise.

\section{Acknowledgement \\ The authors would like to thank Chemi Nutra, Inc. for providing financial and material support of this study. Thanks are also due to the Kilgore Research Center at West Texas A\&M University for providing funding for this study. We would also like to thank the researchers at the Exercise and Sport Nutrition Laboratory at Texas A\&M University for their help in completing this project.}

\section{Author details}

Department of Sport and Exercise Science, West Texas A\&M University, Canyon, TX, 79016, USA. ${ }^{2}$ Increnovo LLC, 2138 E Lafayette PI, Milwaukee, W 53202, USA. ${ }^{3}$ Department of Health and Kinesiology, Texas A\&M University, College Station, TX, 77843, USA.

Published: 15 September 2010

doi:10.1186/1550-2783-7-S1-P2

Cite this article as: Parker et al:: The effects of phosphatidylserine supplementation on cognitive functioning prior and following an acute bout of resistance training in young males. Journal of the International Society of Sports Nutrition 2010 7(Suppl 1):P2. 